\title{
La fonction RH dans les associations : les valeurs militantes à
}

l'épreuve de la professionnalisation

HR management in nonprofits : activist values put to the test of professionalization

\section{Función de recursos humanos en las asociaciones : los valores militantes al examen de la profesionalización}

\section{Patrick Valéau}

Numéro 328, avril 2013

Les coopératives agricoles des « Sud » : quels enseignements pour les "Nord »?

Agricultural Cooperatives in the "South »: What Lessons for the " North »?

URI : https://id.erudit.org/iderudit/1015529ar

DOI : https://doi.org/10.7202/1015529ar

Aller au sommaire du numéro

Éditeur(s)

Association Recma

ISSN

1626-1682 (imprimé)

2261-2599 (numérique)

Découvrir la revue

Citer cet article

Valéau, P. (2013). La fonction RH dans les associations : les valeurs militantes à l'épreuve de la professionnalisation. Revue internationale de l'économie sociale, (328), 76-94. https://doi.org/10.7202/1015529ar
Résumé de l'article

La France compte aujourd'hui 1,1 million d'associations, employant 1,9 million de salariés à temps complet ou partiel. A partir des données de cent quarante d'entre elles, cet article analyse la façon dont la fonction ressources humaines (RH) émerge dans ce contexte, compte tenu des acteurs et des valeurs en présence. Les résultats montrent que même si la structuration des pratiques RH reste en partie liée à la taille des effectifs salariés, elle découle également des choix stratégiques de l'association. Le recrutement par le conseil d'administration d'un directeur gestionnaire apparaît comme le principal déclencheur d'un processus de professionnalisation précédant l'arrivée d'un directeur des ressources humaines (DRH). Plus efficaces et, ce faisant, plus conformes aux attentes des pouvoirs publics et des financeurs, ces nouvelles pratiques RH s'accompagnent cependant d'un déclin du militantisme et des valeurs communautaires. 


\title{
LA FONCTION RH DANS LES ASSOCIATIONS : LES VALEURS MILITANTES À L'ÉPREUVE DE LA PROFESSIONNALISATION
}

par Patrick Valéau*

\begin{abstract}
*Maître de conférences, HDR, IAE de la Réunion, membre du Cemoi. Mél. : patrick@ valeau.com.
\end{abstract}

La France compte aujourd'hui 1,1 million d'associations, employant 1,9 million de salariés à temps complet ou partiel. A partir des données de cent quarante d'entre elles, cet article analyse la façon dont la fonction ressources humaines $(R H)$ émerge dans ce contexte, compte tenu des acteurs et des valeurs en présence. Les résultats montrent que même si la structuration des pratiques $R H$ reste en partie liée à la taille des effectifs salariés, elle découle également des choix stratégiques de l'association. Le recrutement par le conseil d'administration d'un directeur gestionnaire apparaît comme le principal déclencheur d'un processus de professionnalisation précédant l'arrivée d'un directeur des ressources humaines (DRH). Plus efficaces et, ce faisant, plus conformes aux attentes des pouvoirs publics et des financeurs, ces nouvelles pratiques $R H$ s'accompagnent cependant d'un déclin du militantisme et des valeurs communautaires.

\begin{abstract}
HR management in nonprofits : activist values put to the test of professionalization In France today, there are 1.1 million nonprofits employing 1.9 million full- and part-time workers. Using data from 140 nonprofit organizations, this article examines how HR management is formed in this context, taking into account the kinds of organizations and values found in the voluntary sector. Even though the introduction of formal HR practices remains partly tied to the number of employees, the findings show that it also comes from the organization's strategic choices. The recruitment of a managing director by the board appears as the main trigger for a process of professionalization that leads to hiring an HR manager. More effective and consequently more in line with the expectations of government and funding partners, these new HR practices are however accompanied by a decline in activism and community values.
\end{abstract}

Función de recursos humanos en las asociaciones: los valores militantes al examen de la profesionalización

La Francia cuenta actualmente con 1,1 millones de asociaciones empleando 1,9 millones de trabajadores a tiempo completo o parcial. A partir de los datos de 140 de entre ellas, este artículo analiza la manera en que la función de los "Recursos humanos" aparece en este contexto, teniendo en cuenta los actores y los valores presentes. Los resultados muestran que aunque la estructuración de las prácticas de Recursos Humanos queda en parte ligada al tamaño de los efectivos asalariados, ella deriva igualmente de las decisiones estratégicas de la asociación. El reclutamiento, por el consejo de administración, de un director gestionario aparece como 
el principal detonador de un proceso de profesionalización antecediendo la llegada de un Director de Recursos Humanos. Estas nuevas prácticas de Recursos Humanos son ciertamente mas eficaces y más conformes a las expectativas de los poderes públicos y de los financieros, pero ellas se acompañan sin embargo de la decadencia de la militancia y de los valores comunitarios.

Aujourd'hui en France, il existe plus d'un million d'associations, employant 13 millions de bénévoles et 1,9 million de salariés. Près de 270000 d'entre elles créent 1,1 million de postes équivalents temps plein, soit, suivant les estimations, de 6 à $7 \%$ de l'emploi national (Tchernonog, 2007). Les bénévoles demandent une gestion spécifique (Archambault, Prouteau, 2009; Mignonac et al., 2010), mais qu'en est-il des salariés du secteur?

Les partenaires extérieurs, notamment les financeurs, et les bénéficiaires souhaitent souvent que cette ressource soit organisée de façon «professionnelle ». Cependant, en interne, une partie des acteurs reste très attachée aux valeurs militantes, particulièrement celles de liberté et d'égalité. Compte tenu de ces deux contraintes, la gestion des ressources humaines (GRH) des associations est-elle semblable à celle des entreprises? Le développement de la fonction ressources humaines $(\mathrm{RH})$ y suit-il les mêmes étapes? Y est-il aussi principalement fonction de la taille (Fombonne, 2001)? Dans les associations, trois acteurs participent successivement à l'introduction de pratiques $\mathrm{RH}$ : le président bénévole, le directeur salarié et le directeur des ressources humaines (DRH). Ces acteurs amènent-ils un développement progressif ou une suite de transformations ponctuées de crises (Greiner, 1972)? En lien avec "l'éternelle question de la taille» (Draperi, 2012), cet article étudie dans quelle mesure les valeurs militantes du départ peuvent survivre à cette professionnalisation. Suivant une approche scientifique, l'étude de la GRH dans les associations commence par une observation rigoureuse des pratiques dans leur contexte (Pichault, Nizet, 2000; Davister, 2006). Quelques recherches analysent les caractéristiques des emplois créés (Bailly et al., 2012; Tchernonog, 2007) et les motivations de ceux qui les occupent (Combe-Jauret, Lethielleux, 2012). D'autres abordent la question du management: suivant des approches qualitatives (Pichault, Nizet, 2000; Valéau, 2003; Bayle, 2010) ou quantitatives (Saulquin, 2000; Meyer, Ahana, 2010), elles mettent en exergue les tensions possibles entre les salariés professionnels et les bénévoles militant sur le terrain et au sein des organes de gouvernance. Plusieurs auteurs abordent également l'organisation du travail: Dussuet (2010), Richez-Battesti et Oswald (2010) mettent en avant les régulations collectives et les espaces de médiation. Tous ces articles posent un cadre de réflexion dans lequel penser les

(1) Pour une revue complète de ces travaux: Everaere, Valéau, 2012. problématiques de la GRH ${ }^{(1)}$, mais ils n'étudient pas explicitement et systématiquement les contenus de cette dernière: ils n'abordent pas la gestion prévisionnelle des emplois et des compétences (GPEC), la définition des postes, le recrutement, les licenciements, la formation, la paie, etc. La connaissance du fonctionnement des associations demeure très incomplète (Hoarau, Laville, 2008), et les pratiques de ces dernières en matière de GRH restent à analyser de façon plus approfondie. A partir de données recueillies auprès de cent quarante associations, grâce à un questionnaire composé de soixante questions, notre recherche étudie 
la façon dont les pratiques RH émergent dans ce type de contexte. Par " fonction RH », nous entendons l'ensemble des activités destinées à fournir à l'organisation les ressources humaines nécessaires à son développement (Bélanger et al., 1983; Plane, 2000). Nous distinguons, d'un côté, l'organisation et la GPEC visant à articuler les RH aux besoins de l'association et, de l'autre, un management cherchant à acquérir et à dynamiser les RH disponibles (Bélanger et al., 1983; Benchemam, Galindo, 2009). Nous commençons par formuler une série de trois hypothèses fondées sur la confrontation entre la littérature sur les associations et celle sur la GRH. Nous présentons ensuite le dispositif méthodologique mis en place et les résultats obtenus. Nous terminons par une discussion autour des défis de la GRH entre professionnalisation et préservation des valeurs militantes.

\section{Pour une approche scientifique de la GRH dans les associations}

Les écrits sur la GRH dans les associations loi 1901 ont souvent pris la forme de discours prescriptifs relativement contradictoires. D’un côté, sur les questions de stratégie, de finances et de marketing, certains auteurs se tournent vers des modèles issus des entreprises. C'est le cas par exemple de l'entrepreneuriat social. Ce mouvement, initié aux Etats-Unis par Dees (1998) et popularisé en France par Sybille (2010) et la Conférence permanente des coordinations associatives (CPCA, 2010), prend ses racines dans une longue tradition anglosaxonne de manuels prônant une gestion plus efficace. Les chapitres consacrés à la GRH recommandent l'usage de fiches de poste et de systèmes d'incitation et d'évaluation (Brudney, 1994; Herman, 1994). A l'opposé, certains sociologues (Laville, Sainsaulieu, 1997; Chauvière, 2007; Draperi, 2010) dénoncent les excès d'une formalisation risquant, selon eux, d'altérer l'implication des acteurs et les liens communautaires qui les unissent. Pour eux, "trop de gestion » risquerait de «tuer» la dynamique associative (Chauvière, 2007).

Des voix de plus en plus nombreuses renvoient aujourd'hui dos à dos ces deux points de vue, soulignant les limites de discours prescriptifs et normatifs qui s'affrontent sur des questions de principe. De plus en plus d'auteurs en appellent à des approches davantage descriptives et ancrées dans les réalités de la pratique (Pichault, Nizet, 2000; Richez-Battesti, Oswald, 2010; Chemin, Gilbert, 2010).

\section{La littérature scientifique sur la GRH}

Les études de cas qualitatives à base d'observations et d'entretiens semidirectifs sont aujourd'hui relativement nombreuses. Elles rendent compte, en détail, des expériences et des pratiques des acteurs sur le terrain (Laville, Sainsaulieu, 1997). Valéau (2003) décrit les processus d'implication à travers lesquels les individus se lient aux associations qu'ils rejoignent. Védélago et al. (2004) soulignent les possibles tensions entre militants bénévoles et professionnels salariés à propos du projet associatif. Dussuet (2010), Richez-Battesti et Oswald (2010) évoquent également des espaces de discussion, de régulation et de médiation entre les acteurs dans le cadre desquels "se construit et se reconstruit le "faire ensemble" ». Suivant une approche plus transversale, 
Everaere (2011) relève un certain nombre de liens possibles entre les valeurs indiquées dans des discours et les pratiques effectivement mises en place. Bayle (2010), Boncler et Valéau (2010) constatent cependant la place que finissent souvent par prendre les salariés dans la gouvernance de ces organisations. Ces différentes études montrent chacune à sa façon comment les interactions entre les parties prenantes structurent les associations. Selon l'Association pour le développement de la documentation sur l'économie sociale (www.addes.asso.fr), il n'y aura "pas d'avancée sans recherche ni chiffres». Les études statistiques des auteurs affiliés à cette association, tels que Tchernonog (2007) et Kaminski (2006), intègrent des éléments de GRH en lien avec les profils des dirigeants bénévoles et les caractéristiques des emplois salariés créés, mais restent à vocation macroéconomique. Les chiffres relatifs à ce qui se passe à l'intérieur des associations restent rares. Suivant une approche psychosociologique classique, Meyer et Ohana (2010) mesurent les effets de la justice interactionnelle sur la cohésion de groupe et les comportements citoyens. Saulquin (2000) montre que la participation des salariés aux décisions et la prise en compte par le directeur de leurs performances contribuent à la qualité du service. Ces études confirment les explorations qualitatives concernant l'importance des valeurs dans les logiques d'action des acteurs.

Les sociologues et les économistes contribuent à l'étude du fonctionnement des associations, mais, comme le relevaient récemment Hoarau et Laville (2008), la gestion des associations demeure encore, en tant que telle, " un angle mort». Ces études qualitatives et quantitatives que nous venons d'évoquer posent un cadre général permettant d'aborder certains aspects des ressources humaines, mais elles restent très incomplètes au regard des contenus définis par la recherche en gestion. Il convient donc de repartir des définitions de la GRH pour étudier les phénomènes en question dans le contexte associatif.

Les recherches et les manuels de GRH évaluent le degré de développement de la fonction RH à travers la nature et la qualité des activités mises en place. Ils distinguent une simple administration du personnel d'une véritable gestion des ressources humaines. La première commence avec les recrutements, les rémunérations et les assignations (Bélanger et al., 1983; Louart, 1990; Fombonne, 2001). Les activités ensuite mises en place concernent l'organisation et la mobilisation. L'organisation relie la fonction RH aux besoins découlant de la stratégie de l'entreprise (Bélanger et al., 1983; Peretti, 2009). Elle consiste à rationaliser le travail, en définissant successivement la structure, les postes, les règles et les procédures (Peaucelle, 2003; Thévenet et al., 2009). Elle aboutit à une GPEC (Plane, 2000) s'appuyant sur des systèmes d'information plus sophistiqués (Le Louarn, 2008). La seconde série d’activités reprise par l'ensemble des manuels a trait à la mobilisation des RH disponibles. Ces pratiques de développement des compétences et des motivations sont souvent qualifiées de progressives (Delaney, Huselid, 1996; Thévenet et al., 2009). Elles recouvrent la gestion des carrières en lien avec l'évaluation, la formation et l'individualisation des rémunérations. La fonction $\mathrm{RH}$ a pour but principal de fournir à l'organisation le travail nécessaire à la réalisation des objectifs de celle-ci. Elle doit lui permettre de réaliser le niveau et la qualité de production visés, tout en minimisant les coûts (Martory, Crozet, 1998; Péretti, 2009). 
La recherche sur les associations n'a pas encore véritablement étudié les aspects administratifs, techniques et organisationnels de la fonction $\mathrm{RH}$. Pichault et Nizet (2000), Boncler et Valéau (2010) et Everaere (2011) ont certes donné quelques exemples de pratique, mais le recrutement, le licenciement, la paie, la définition des postes, la GPEC et les systèmes d'information peuvent être étudiés de façon plus complète et plus systématique dans le cadre de recherches quantitatives. Pour reprendre les termes de Chemin et Gilbert (2010), ces sujets ont trait aux «équipements » et aux « instruments » de la GRH. Ces aspects techniques n'ont jusque-là suscité que peu d'intérêt, certains les trouvant sans doute trop éloignés des grandes questions de stratégie et de gouvernance. Pourtant, si ces outils peuvent être «façonnés » en fonction du projet stratégique (Richez-Battesti, Oswald, 2010), ils peuvent aussi avoir un «effet politique» (Chemin, Gilbert, 2010).

\section{Hypothèses sur l'émergence de la fonction RH}

L'objectif de cette recherche est d'apporter une série d'éléments empiriques nouveaux sur la façon dont les pratiques RH sont implémentées dans le contexte associatif. Suivant les approches contingentes, celles-ci doivent être adaptées aux caractéristiques technico-économiques et sociopolitiques de leurs environnements interne et externe (Pichault, Nizet, 2000; Mercier, Schmidt, 2004). La GRH devient, ce faisant, une "création sous contraintes " (Louart, 1990). Nos hypothèses confrontent donc la littérature sur la GRH à celle sur le contexte associatif.

\section{PREMIÈRE HYPOTHÈSE : LE DÉVELOPPEMENT DES PRATIQUES RH EST LIÉ AUX EFFECTIFS SALARIÉS}

Pour la littérature sur la GRH, le développement de la fonction est directement lié à la taille de l'entreprise (Fombonne, 2001). Greiner (1972) montre que les modes de supervision mis en place au départ trouvent leurs limites face à des effectifs plus importants. Pour cet auteur, la première crise traversée par les organisations en croissance concerne le management et aboutit au stade de la direction caractérisé par une structure plus fonctionnelle, des profils de poste plus formels et des procédures de travail plus standardisées. Pour les tenants de la gestion et de l'entrepreneuriat social, l'association reste une organisation presque comme les autres (Herman, 1994; Dees, 1998; Brudney, 1994). Everaere (2011) relie également une partie de ses observations sur les petites associations à la GRH dans les très petites entreprises (TPE) : une GRH opportuniste, intuitive, voire improvisée. Notre première hypothèse est donc que la fonction RH dans les associations n'échappe pas aux contraintes de coordination et de centralisation: son développement serait, comme dans les entreprises, en partie induit par la croissance des effectifs.

\section{DEUXIĖME HYPOTHĖSE : LE DÉVELOPPEMENT DES PRATIQUES RH DÉPEND DE LA CRÉATION D'UN POSTE DE GESTIONNAIRE, PUIS D'UN POSTE SPÉCIALISÉ DANS LA GRH}

La littérature sur la GRH, en particulier les manuels, commence souvent par interroger le niveau d'émergence de la fonction RH. Pour Louart (1990), l'absence de responsable des ressources humaines ne signifie pas l'inexistence 
de la fonction, elle indique simplement un faible degré de formalisation. Il existe une GRH de fait, dès lors que l'organisation emploie un salarié (Fombonne, 2001; Louart, 1990). Cela dit, la création d'un service autour d'un ou de plusieurs spécialistes du domaine marque une étape importante dans le développement de la fonction. Elle signifie le début d'une coordination rationnelle de ces activités (Barraud et al., 2004; Mercier, Schmidt, 2004; Péretti, 2009; Thévenet et al., 2009). Un tel service apparaît généralement entre cent et deux cent cinquante salariés (Fombonne, 2001). Selon la littérature sur la GRH (Fombonne, 2001; Barraud et al., 2004), le développement de la fonction $\mathrm{RH}$ requiert des compétences spécifiques. La littérature sur les associations note un manque de compétences des gestionnaires bénévoles (Davister, 2006; Mayaux, 1996). Saulquin (2000) relevait le rôle joué par les directeurs d'établissement médicosocial en matière de GRH. Bayle (2010), Boncler et Valéau (2010) et Védélago et al. (2004) soulignaient également l'ascendant que pouvaient prendre les professionnels salariés sur les dirigeants bénévoles. Notre deuxième hypothèse est donc que, au-delà des effectifs, le recrutement de professionnels salariés compétents en GRH favorise l'introduction de nouvelles pratiques dans le cadre de stratégies plus abouties.

\section{TROISIĖME HYPOTHĖSE : LE DÉVELOPPEMENT DES PRATIQUES RH FAIT ÉVOLUER LES VALEURS DE L'ASSOCIATION}

Les associations obligent à repenser partiellement la gestion en général et la GRH en particulier compte tenu, notamment, de la place occupée par les valeurs (Davister, 2006; Rousseau, 2007; Richez-Battesti, Oswald, 2010; Valéau, 2003). Boncler et Valéau (2010) lient le mouvement associatif français aux valeurs de fraternité, de liberté et d'égalité. Celles-ci se retrouvent, sous différentes formes, dans la plupart des définitions des organisations de l'économie sociale et solidaire. Ainsi, la charte européenne de l'économie sociale, la définition de l'Emes (Defourny, Nyssens, 2008 et 2011) ou celle retenue par Salamon et Anaheier (1997) pour leur étude internationale retiennent toutes la priorité donnée à un projet d'utilité sociale (fraternité), l'autonomie de gestion (liberté) et le principe d'une gouvernance relativement participative (égalité). Pour Everaere (2011), l'amélioration du sort de l'humain constitue le but même des organisations de l'ESS. Boncler et Valéau (2010) relient ces valeurs à la GRH: la fraternité se retrouve généralement dans les motivations des salariés; la liberté inspire un management a priori plus participatif, leur offrant davantage d'autonomie; l'égalité touche à la dimension démocratique des associations et, ce faisant, à l'éventuelle participation des salariés aux décisions.

Le respect des valeurs prend, dans les associations, une importance parfois égale ou supérieure à la réalisation des finalités. Le principe de propriété s'effaçant au profit d'une agora de parties prenantes qui participent à leur gouvernance (Boncler, Valéau, 2010), les questions de légitimité deviennent aussi primordiales que la rationalisation des moyens mis en œuvre (Biondy et al., 2010). La gestion des associations passe ainsi d'un paradigme fondé sur la maximisation sous contrainte à une gestion multicritères faite de contradictions, d'équilibrages, mais aussi d'arbitrages (Valéau, 2003). Everaere (2011) avait repéré, dans le cadre d'un échantillon de vingt-cinq organisations, un certain nombre de cas illustrant les liens possibles entre 
les valeurs affichées et les pratiques $\mathrm{RH}$, avec par exemple des écarts de rémunération plus réduits, des recrutements plus indulgents ou une gestion plus autonome des temps de travail.

Mais compte tenu de la primauté qu'elles accordent aux valeurs et à l'humain, les contradictions entre l'efficacité économique et le social qui caractérisent les GRH (Brabet, 1993; Louart, 1990) se retrouvent, au sein des associations, plus fortes que jamais (Valéau, 2003). Des tensions peuvent apparaître entre des pratiques $\mathrm{RH}$ fondées sur une coordination et des évaluations de type hiérarchique et les principes de liberté, d'égalité et de collectivité auxquels les associations sont souvent attachées (Boncler, Valéau, 2010). Chemin et Gilbert (2010) montrent que, derrière leur apparente neutralité, les instruments de gestion ont un effet politique susceptible de transformer en profondeur les associations. La montée en puissance des salariés professionnels (Bayle, 2010; Boncler et Valéau 2010; Védélago et al., 2004) se traduit par l'introduction de nouvelles pratiques, mais aussi de nouvelles valeurs.

\section{Méthodes}

Cette recherche a été menée en collaboration avec la chambre régionale de l'économie sociale (Cres) de la Réunion. La population ciblée était celle des associations employant au moins un salarié. Cent quarante des 1500 associations employeurs contactées nous ont retourné notre questionnaire dûment rempli, soit un taux de réponse de $9,33 \%$. Les associations comprenant un ou deux salariés étaient moins représentées que dans la population mère (Tchernonog, 2007), alors que celles de plus de vingt étaient plus nombreuses (tableaux 1 et 2 en page suivante). Nous disposions ainsi d'échantillons par catégorie suffisants pour observer les changements de pratiques liés à la taille des effectifs.

Le questionnaire créé pour l'occasion comprenait soixante items, dont quarante utilisés dans le cadre de cette recherche. La première partie portait sur les activités et les effectifs de l'association ainsi que sur les personnes impliquées dans la GRH. La deuxième concernait la définition des besoins, la gestion des emplois et des compétences et les fiches de poste. La troisième étudiait les moyens mis en œuvre pour adapter les RH disponibles,

\section{Tableau 1}

\section{Structure de l'échantillon par effectif salarié}

\begin{tabular}{|c|c|c|c|c|c|c|c|c|c|}
\hline & 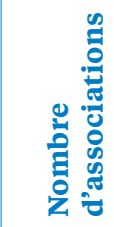 & $\dot{\sum}$ & 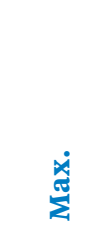 & $\dot{\vec{c}}$ & 苞 & $\underset{\pi}{\pi}$ & 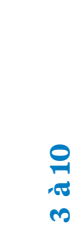 & $\begin{array}{l}\text { 옥 } \\
\text { స్ }\end{array}$ & $\frac{\mathscr{O}}{\stackrel{\Xi}{\Xi}}$ \\
\hline $\begin{array}{l}\text { Effectif } \\
\text { salarié }\end{array}$ & 140 & 1 & 450 & 17,44 & 42,87 & \begin{tabular}{|l|}
35 \\
$25 \%$ \\
$(44 \%)$
\end{tabular} & $\begin{array}{l}56 \\
40 \% \\
(30 \%)\end{array}$ & $\begin{array}{l}25 \\
17 \% \\
(24 \%)\end{array}$ & $\begin{array}{l}24 \\
18 \% \\
(2 \%)\end{array}$ \\
\hline
\end{tabular}

Entre parenthèses, la population mère (Tchernonog, 2007). 


\section{Tableau 2}

Structure de l'échantillon par secteur d'activité*

\begin{tabular}{|l|l|l|l|l|l|l|}
\hline Secteurs & Education & Santé & Social & Sport & Culture & Loisir \\
\hline & $28 \%$ & $9 \%$ & $27 \%$ & $14 \%$ & $12 \%$ & $10 \%$ \\
\hline
\end{tabular}

* Le secteur des ONG humanitaires n'était pas représenté.

notamment les recrutements, les licenciements et la formation. Quelques questions avaient pour sujet les rémunérations, en particulier les primes. La dernière partie abordait le thème des valeurs, notamment les oppositions entre liberté et directivité, entre égalité et hiérarchie, le partage des objectifs et des valeurs. Les questions posées relevaient davantage des opinions et des

(2) L'analyse en composantes principales est une méthode qui permet de structurer un ensemble des variables (ici despratiques RH) en quelques grands groupes, aussi appelés « axes".

(3) Un modèle de régression linéaire est un modèle mettant en relation une variable expliquée et une ou plusieurs variables explicatives. ressentis, par exemple: «Existe-il une véritable hiérarchie au sein de votre organisation à but non lucratif? »; "Souhaitez-vous que vos salariés adhèrent aux buts et aux valeurs de votre association?»; "Dans les réunions, vos salariés peuvent-ils émettre des opinions opposées à celles de la direction?» Afin d'optimiser les possibilités d'analyse, la plupart des questions étaient assorties d'échelles continues allant de 1 à 7. Les statistiques descriptives (moyennes et écarts types), les analyses en composantes principales ${ }^{(2)}$, les corrélations bilatérales, les différences des moyennes et les régressions linéaires ${ }^{(3)}$ ont été réalisées à l'aide du logiciel SPSS. Les analyses quasi implicatives (voir infra) ont été effectuées avec le logiciel Chic (Couturier et al., 2005).

\section{Résultats}

La présentation de nos résultats commence par une analyse des pratiques RH. Nous identifions ensuite les liens entre ces dernières et les acteurs impliqués dans la fonction RH. Nous terminons avec les relations entre ces deux séries de variables et les valeurs des associations.

\section{L’introduction de pratiques RH plus élaborées}

Les analyses en composantes principales des pratiques RH mettent au jour leur structure, tout en réduisant le nombre de variables par la suite étudiées. Suivant la dichotomie introduite par la littérature sur la GRH, nous analysons tour à tour l'organisation des RH et la mobilisation (tableau 3, en page suivante).

La première composante de l'organisation des RH met en lien les informations et les prévisions liées à la GPEC. La deuxième composante rassemble les expertises relatives au droit du travail et au financement de la formation. La troisième a trait à la définition des postes.

Concernant la mobilisation, la première composante associe les promotions et les licenciements. Elle constitue une forme de gestion des carrières faisant évoluer les individus ou s'en séparant en fonction des besoins de l'organisation. La deuxième correspond à l'introduction des évaluations. La troisième, intitulée «Investissements RH », associe les CDI, la formation, les systèmes de rémunération incitatif. 


\section{Tableau 3}

Analyses en composantes principales des pratiques $\mathrm{RH}$

\begin{tabular}{|c|c|c|c|c|c|c|c|}
\hline \multicolumn{4}{|c|}{ Organisation des RH } & \multicolumn{4}{|c|}{ Mobilisation des RH } \\
\hline & 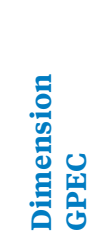 & 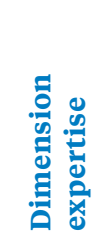 & 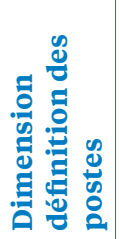 & & 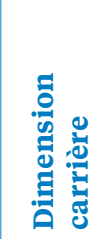 & 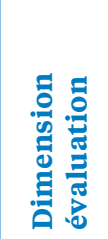 & 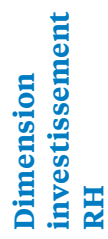 \\
\hline Profil de poste & 0,132 & 0,224 & 0,718 & $\% \mathrm{CDI}$ & 0,213 & $-0,165$ & 0,653 \\
\hline Fiche de poste & 0,100 & $-0,051$ & 0,827 & Evaluation & 0,035 & 0,900 & 0,015 \\
\hline Prévision activité & 0,828 & $-0,137$ & 0,140 & $\begin{array}{l}\text { Entretiens } \\
\text { annuels }\end{array}$ & 0,032 & 0,875 & 0,129 \\
\hline $\begin{array}{l}\text { Prévision RH } \\
\text { disponibles }\end{array}$ & 0,825 & 0,222 & 0,101 & Promotion & 0,545 & 0,052 & 0,444 \\
\hline Tableaux de bord & 0,741 & 0,182 & 0,079 & Primes & $-0,126$ & 0,142 & 0,805 \\
\hline Logiciels RH & 0,396 & 0,470 & 0,158 & Formation & 0,213 & 0,162 & 0,592 \\
\hline Connaissance OPCA & $-0,134$ & 0,806 & 0,100 & $\begin{array}{l}\text { Licenciement } \\
\text { économique }\end{array}$ & 0,759 & $-0,026$ & 0,125 \\
\hline Droit du travail & 0,250 & 0,679 & $-0,001$ & $\begin{array}{l}\text { Licenciement } \\
\text { disciplinaire }\end{array}$ & 0,702 & 0,070 & $-0,017$ \\
\hline Alpha de Cronbach & 0,75 & 0,42 & 0,42 & & 0,49 & 0,77 & $\mathbf{0 , 5 4}$ \\
\hline
\end{tabular}

Organisation des RH/Varimax quatre itérations. $62 \%$ variance restituée, $\mathrm{KMO}=0,68$; Bartlet < 0,000.

Management/Varimax cinq itérations. $72 \%$ variance restituée, $\mathrm{KMO}=0,5$; Bartlet $<0,000$.

Les alphas de Cronbach - indice le plus couramment utilisé pour évaluer la consistance d'une échelle - des dimensions GPEC et évaluation sont très satisfaisants. Ceux des dimensions expertise, définition des postes, carrière et investissement RH apparaissent, en revanche, relativement faibles. Nous conservons ces dimensions compte tenu du caractère exploratoire de notre recherche.

La figure 1 (en page suivante) dessine deux " grappes de pratiques »(Delaney, Huselid, 1996). La première concerne les besoins RH. Elle commence par la définition de poste susceptible d'être utilisée dans le cadre d'évaluations des compétences. La mesure des écarts entre les deux permet la mise en place d'une GPEC. Le deuxième bloc, relatifà la gestion des RH disponibles, démarre avec des expertises fiscales et juridiques permettant d'investir des pratiques plus élaborées, comme la formation, susceptibles d'aboutir à une véritable gestion des carrières. Cette deuxième grappe est globalement conditionnée au développement de la première. Les pratiques composant la grappe « $\mathrm{RH}$ disponibles " sont significativement corrélées à la taille de l'effectif (tableau 4, en page suivante). Elles confirment notre première hypothèse. Celles de la grappe « Besoins RH » restent, en revanche, relativement indépendantes de cette variable. Elles contredisent cette hypothèse. 


\section{Figure 1}

Analyse quasi implicative entre les différentes dimensions de la GRH

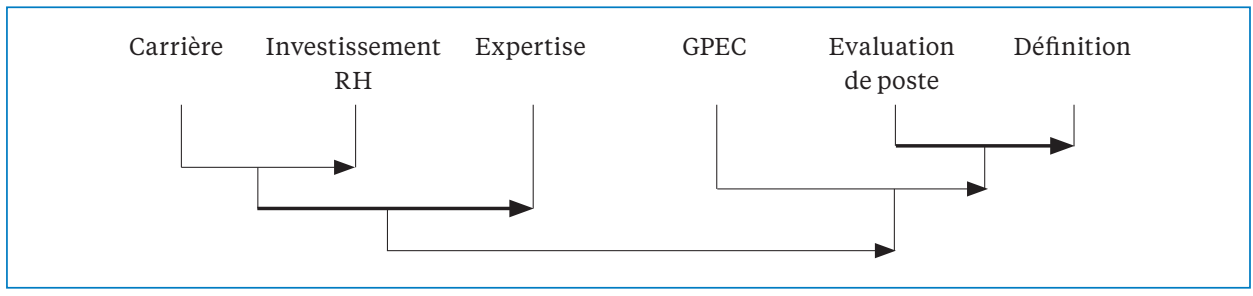

Tableau 4

Corrélation de Pearson entre les différentes dimensions de la GRH

\begin{tabular}{|c|c|c|c|c|c|c|}
\hline & 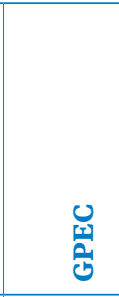 & 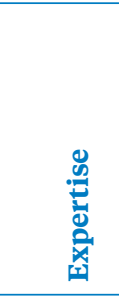 & 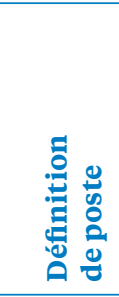 & 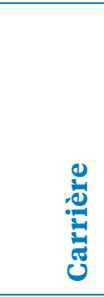 & 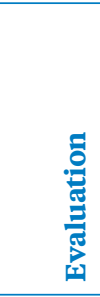 & 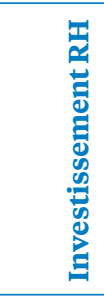 \\
\hline GPEC & - & & & & & \\
\hline Expertise & $0,177^{*}$ & - & & & & \\
\hline $\begin{array}{l}\text { Définition } \\
\text { de poste }\end{array}$ & $0,283^{* *}$ & $0,198^{*}$ & - & & & \\
\hline Carrière & 0,143 & $0,305^{* *}$ & 0,147 & - & & \\
\hline Evaluation & $0,215^{*}$ & 0,109 & $0,422^{* *}$ & 0,102 & - & \\
\hline Investissement & $0,177^{*}$ & $0,404^{* *}$ & 0,119 & $0,356^{* *}$ & $0,191^{*}$ & - \\
\hline Effectif & 0,121 & $0,267^{* *}$ & 0,057 & $0,515^{* *}$ & 0,082 & $0,294^{* *}$ \\
\hline
\end{tabular}

** $=\operatorname{sig} 0.01 ;{ }^{*}=0.05$.

La méthode consiste à établir la probabilité d'une dimension de la GRH en fonction d'une autre. Les flèches de la figure 1 signifient "si $A$, alors $B$ », sans figurer la réciproque. Cela permet, à la différence des corrélations, de pouvoir donner une direction à la relation et d'interpréter $\mathrm{B}$ comme un passage quasi obligé pour le développement de A. Par exemple, la définition de poste constitue une condition plus ou moins nécessaire pour envisager une évaluation. En d'autres termes, si l'association met en place une évaluation, il est fort probable qu'elle ait en amont une fiche de poste; par contre, elle peut très bien avoir mis en place un tel document, mais ne pas évaluer.

\section{Professionnalisation et évolution des pratiques}

Conformément à notre deuxième hypothèse, le développement des pratiques RH apparaît significativement différent en fonction des acteurs présents (tableau 5, en page suivante). La probabilité d'une différence 


\section{Tableau 5}

\section{Différences des moyennes entre les pratiques du président, du directeur et du DRH}

\begin{tabular}{|c|c|c|c|c|c|c|c|c|}
\hline & U్ & 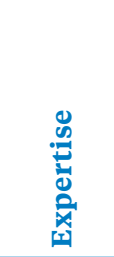 & 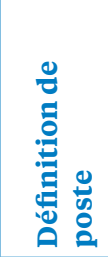 & 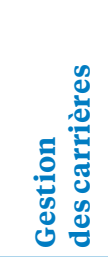 & 苞 & 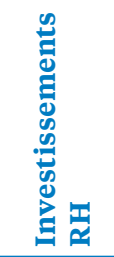 & 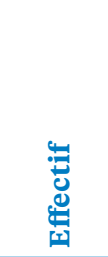 & 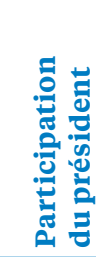 \\
\hline $\begin{array}{l}\text { Président } \\
\text { /Directeur }\end{array}$ & $-2,24^{*}$ & $-4,27^{* *}$ & $-2,38^{*}$ & $-3,76^{* *}$ & $-1,30$ & $-6,95^{* *}$ & $-4,81^{* *}$ & $4,28^{* *}$ \\
\hline $\begin{array}{l}\text { Directeur } \\
\text { /DRH }\end{array}$ & $-1,32$ & 0,82 & 1,39 & $-3,05^{*}$ & $-2,14^{*}$ & 0,51 & $-3,17^{* *}$ & 0,43 \\
\hline
\end{tabular}

$t$ de Student: ${ }^{* *}=\mathrm{s}$ ig $0.01 ;^{*}=0.05$.

\section{Figure 2}

\section{Structuration de la fonction $\mathrm{RH}$}

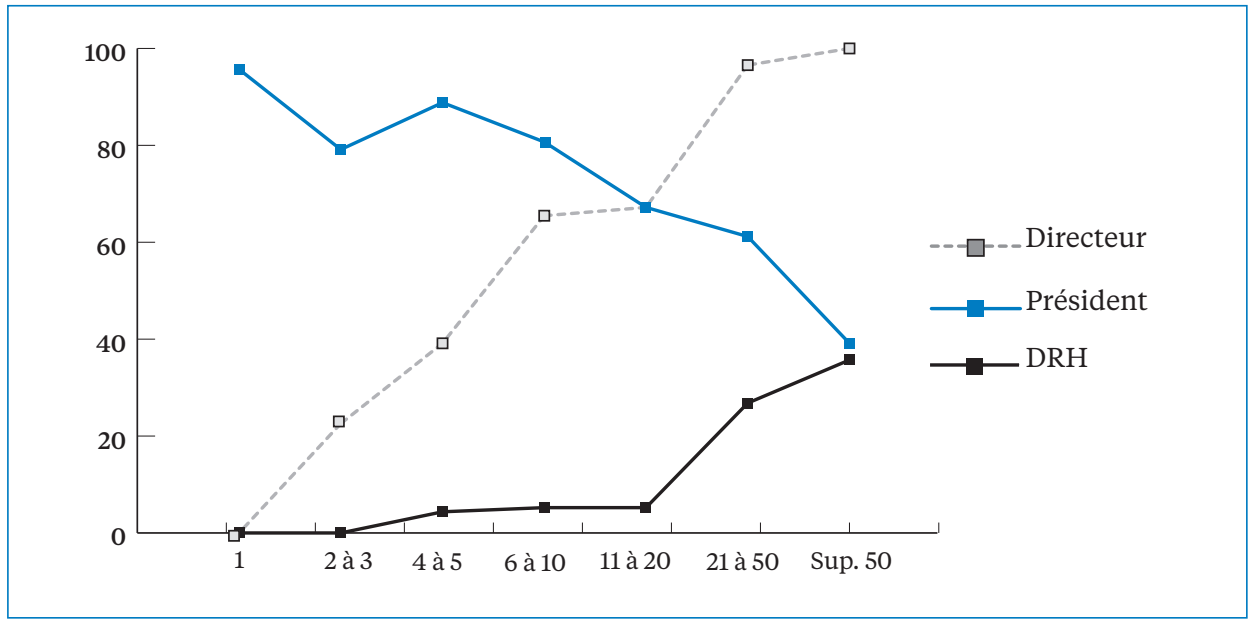

des moyennes entre le directeur et le président est supérieure à $95 \%$ pour cinq des six dimensions de la GRH: la GPEC, l'expertise, la définition de poste, la gestion des carrières et les investissements RH. Le DRH achève la gestion des carrières et introduit de façon plus spécifique des systèmes d'évaluation.

Le recrutement d'un directeur, puis d'un DRH reste très lié à la taille de l'effectif (tableau 5). Cependant, comme le montre la figure 2 (ci-dessus), les associations comptant entre deux et vingt salariés sont très partagées sur le recrutement d'un directeur (entre 22 et $76 \%$ ), et celles possédant un effectif supérieur à vingt sont $25 \%$ à avoir recruté un responsable RH. Le directeur vient, au départ, soutenir l'action du président, mais son arrivée semble irrémédiablement diminuer sa participation à la gestion de l'association. 


\section{Emergence de la fonction $\mathrm{RH}$ et évolution des valeurs}

La taille des effectifs ne semble pas avoir d'effet direct sur les valeurs associatives : aucune des deux séries de régressions présentées dans le tableau 6 n'indiquent de bêtas significatifs. Conformément à notre troisième hypothèse, les valeurs apparaissent, en revanche, liées à la professionnalisation des dirigeants et aux pratiques qu'ils introduisent (tableau 7, en page suivante, voir infra). Le temps du président est marqué par le bénévolat, l'informel et l'égalité entre les membres. Celui du directeur introduit des notions plus managériales d'expression, d'initiative et de compétences mesurées en termes de diplôme.

Concernant les pratiques RH (tableau 6), six régressions sur douze sont ajustées pour ce qui est de l'organisation et deux sur douze pour la mobilisation. Les résultats soulignés correspondent aux pratiques $\mathrm{RH}$ jouant un rôle significatif sur les valeurs considérées. De façon logique, la GPEC réduit l'informel communautaire ${ }^{(4)}$, et les fiches et les expertises, les égalités, en

(4) L'« informel communautaire" est une valeur qui consisteàvaloriser les rapports directs et personnels entre les membres plutôt que des systèmes de règles. les remplaçant par une hiérarchie fondée sur les diplômes qui font des RH un moyen. Notons que les fiches de poste peuvent également servir pour les bénévoles et formalisent, paradoxalement, des espaces d'initiative. Les pratiques de management semblent moins impacter les valeurs. Seule l'évaluation remet en question l'informel, avec en contrepartie davantage de possibilités d'expression.

\section{Tableau 6}

\section{Régressions pratiques $\mathrm{RH}$ /valeurs}

\begin{tabular}{|c|c|c|c|c|c|c|c|c|c|c|c|c|}
\hline & 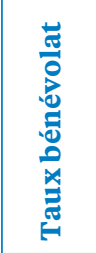 & 总 & 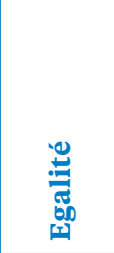 & 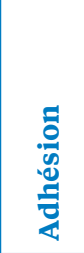 & 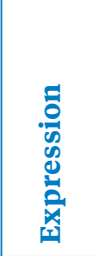 & 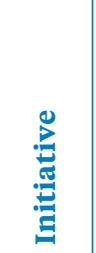 & 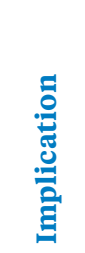 & 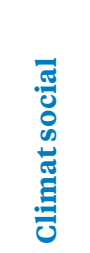 & 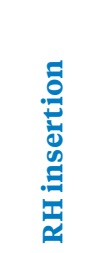 & 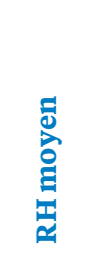 & 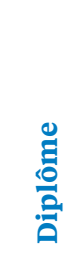 & ஜ્ઞ \\
\hline Effectif & $-0,08$ & $-0,05$ & $-0,05$ & $-0,08$ & 0,06 & 0,01 & 0,04 & $-0,08$ & $-0,01$ & $-0,03$ & 0,12 & 0,06 \\
\hline GPEC & $-0,18$ & $-0,25^{* *}$ & $-0,07$ & $-0,03$ & $-0,04$ & 0,12 & 0,10 & 0,02 & $-0,06$ & $-0,04$ & $0,20^{*}$ & $-0,07$ \\
\hline Expert & $-0,07$ & $-0,16$ & $-0,26^{* *}$ & 0,01 & 0,10 & $-0,06$ & $-0,06$ & $-0,08$ & $-0,23 *$ & 0,16 & 0,1 & $-0,09$ \\
\hline Fiche & $0,20^{*}$ & $-0,13$ & $-0,19 *$ & $0,25^{* *}$ & 0,17 & $0,24^{* *}$ & 0,15 & 0,12 & 0,11 & $0,24^{*}$ & 0,15 & $0,22^{*}$ \\
\hline $\mathbf{F}$ & $2,42^{*}$ & $5,28 * *$ & $\mathbf{5 , 5 7 * *}$ & $2,0 \mathrm{~ns}$ & $1,5 \mathrm{~ns}$ & $2,8^{*}$ & $1,2 \mathrm{~ns}$ & $0,9 \mathrm{~ns}$ & $1,3 \mathrm{~ns}$ & $2,3^{*}$ & $4,6^{* *}$ & $1,4 \mathrm{~ns}$ \\
\hline $\mathbf{R}^{2}$ ajusté & 0,04 & 0,12 & 0,13 & - & - & 0,05 & - & - & - & 0,06 & 0,11 & - \\
\hline Effectif & $-0,14$ & $-0,03$ & $-0,06$ & $-0,10$ & $-0,05$ & $-0,03$ & 0,08 & $-0,16$ & 0,32 & $-0,03$ & 0,15 & 0,00 \\
\hline Carrière & 0,09 & $-0,13$ & $-0,02$ & 0,04 & 0,16 & 0,11 & $-0,10$ & 0,11 & $-0,23$ & 0,09 & 0,04 & 0,16 \\
\hline Evaluation & 0,15 & $-0,19 *$ & $-0,06$ & 0,12 & $0,18^{*}$ & 0,10 & 0,06 & 0,19 & 0,19 & 0,16 & 0,13 & 0,15 \\
\hline Investissement & $-0,16$ & $-0,04$ & $-0,15$ & $-0,01$ & 0,10 & $-0,10$ & 0,07 & $-0,00$ & $-0,11$ & $-0,04$ & $-0,03$ & $-0,20$ \\
\hline $\mathbf{F}$ & 2,07 & $2,78^{*}$ & 1,53 & 0,7 & 2,93 & 0,74 & 0,51 & 1,9 & 2,35 & 0,96 & 1,67 & 2,00 \\
\hline$R^{2}$ ajusté & - & 0,05 & - & - & 0,06 & - & - & - & - & - & - & - \\
\hline
\end{tabular}

$t$ de Student $:^{* *}=\operatorname{sig} 0.01 ;^{*}=0.05$. 


\section{Tableau 7}

Différences des moyennes dirigeants/valeurs

\begin{tabular}{|c|c|c|c|c|c|c|c|c|c|c|c|c|}
\hline & 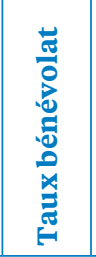 & 을 & 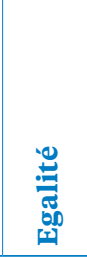 & 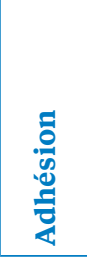 & 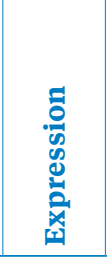 & 莺 & 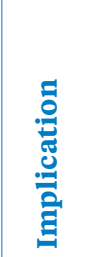 & 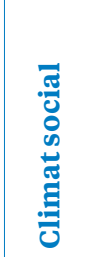 & 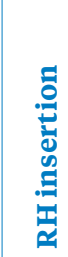 & 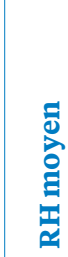 & $\frac{\stackrel{0}{\Xi}}{\stackrel{0}{\circ}}$ & 番 \\
\hline $\begin{array}{l}\text { Président } \\
\text { /Directeur }\end{array}$ & $3,43^{* *}$ & $3,5^{* *}$ & $2,78^{* *}$ & $-0,50$ & $-2,23^{* *}$ & $-0,93^{* *}$ & $-0,62$ & $-0,12$ & 0,12 & 0,27 & $-2,3^{* *}$ & 1,47 \\
\hline $\begin{array}{l}\text { Directeur } \\
\text { /DRH }\end{array}$ & & 0,39 & 0,09 & 0,22 & $-0,88$ & 0,4 & $-0,08$ & 0,33 & 0,85 & 1 & 0,11 & $0,59 * *$ \\
\hline
\end{tabular}

$t$ de Student: ${ }^{* *}=\operatorname{sig} 0.01 ;^{*}=0.05$.

\section{Discussion}

Les résultats obtenus confirment notre première hypothèse: l'émergence de la fonction RH dans les associations dépend pour partie, comme dans les entreprises, de la taille de l'effectif. Cependant, au-delà de ce facteur, nos données mettent surtout en évidence, conformément à notre deuxième hypothèse, le rôle joué par les gestionnaires professionnels: le DRH, mais aussi, bien avant son arrivée, le directeur. La décision de recruter ce dernier intervient de façon relativement variée entre deux et vingt salariés, et son arrivée coïncide généralement avec l'émergence de nouvelles pratiques de GRH. Comme supposé dans la troisième hypothèse, les changements dans l'organisation de la fonction RH tendent à modifier les valeurs communautaires et militantes des débuts, les liens sociaux laissent place à une hiérarchie plus formelle et plus fonctionnelle. Cette discussion restitue les résultats dans le cadre des débats actuels sur les spécificités de la gestion des associations (Biondy et al., 2010; Bayle, 2010). La croissance fait souvent basculer les associations vers une professionnalisation où les questions de " rationalisation » l'emportent sur celles de « légitimité ». Plus précisément, elle introduit d'autres formes de légitimité, différentes de celles définies par les valeurs militantes. Nous questionnons ici les possibilités de défendre ces dernières de façon à préserver une certaine spécificité associative.

\section{Un processus de professionnalisation semblable à d'autres organisations}

De manière presque mécanique, quoi qu'en pensent les acteurs, la taille de l'effectif dans les associations comme dans les entreprises influence grandement le développement des pratiques RH (Fombonne, 2001; Greiner, 1972). Son importance joue en termes d'opportunité: certaines pratiques telles que la gestion des carrières et certaines spécialisations requièrent un organigramme composé de multiples niveaux hiérarchiques. La taille 
de l'effectif introduit aussi de nouveaux besoins, un effectif plus nombreux demandant davantage d'organisation avec des formes de délégation et de coordination plus structurées (Greiner, 1972; Peaucelle, 2003). Ce premier constat ne signifie pas forcément que les associations soient semblables aux entreprises, mais simplement que, malgré leurs spécificités, elles restent aussi des organisations à part entière.

Cette recherche montre les différents rôles successivement joués par les dirigeants: le président, le directeur et le DRH, en tant qu'entrepreneurs de la fonction $\mathrm{RH}$. Le rythme de leur succession modère ou, au contraire, accélère les effets de la croissance sur la professionnalisation. Le temps du président, précédant l'arrivée d'un directeur, est celui de la " communauté militante ». Il donne à l'association, avec l'aide de bénévoles et de salariés, une première impulsion fraternelle et informelle, mais néanmoins entrepreneuriale. A ce stade, la GRH des associations correspond relativement bien à l'idée que s'en font les sociologues: des ajustements mutuels fondés sur le partage des valeurs dans le cadre de liens communautaires (Laville, Sainsaulieu, 1997). Ce modèle peut apparaître comme une absence de gestion. Il constitue en fait un fonctionnement adapté aux valeurs, aux besoins et aux ressources de la situation (Fombonne, 2001; Louart, 1990; Pichault, Nizet, 2000). C'est dans le cadre de cet informel communautaire que se constituent les espaces de médiation évoqués par Dussuet (2010), Richez-Battesti et Oswald (2010). Le temps du directeur ne signifie pas toujours une « désimplication » du président, des binômes peuvent en effet fonctionner (Mayaux, 1996), mais nos résultats indiquent que ce cas de figure n'est pas le plus fréquent. Le temps du directeur est celui de la professionnalisation. Il formalise et structure la fonction RH en introduisant des fiches de poste, des éléments de GPEC, de gestion des carrières, dans le cadre d'une application plus stricte du droit du travail. Saulquin (2000) relevait que $80 \%$ des directeurs des établissements sociaux considéraient la GRH comme un aspect clé de leur fonction. Le DRH complète ensuite l'action du directeur en ce qui concerne la gestion des carrières et introduit des pratiques d'évaluation plus poussées. Son recrutement, entre cinquante et cent cinquante salariés (Fombonne, 2001), constitue davantage un aboutissement qu'une cause de l'émergence de la fonction $\mathrm{RH}$. Ces résultats confirment la montée en puissance des professionnels (Bayle, 2010; Boncler, Valéau, 2010; Védélago et al., 2004). Cette action des directeurs et des DRH n'est pas sans rappeler ce qui se passe au sein des petites et moyennes entreprises à la suite de la phase entrepreneuriale (Everaere, 2011; Greiner, 1972; Robichaud et al., 2005), mais la transformation apparaît encore plus radicale dans le contexte associatif, tant les principes de la professionnalisation diffèrent des valeurs militantes du départ.

\section{La question spécifique des valeurs militantes}

Conformément à la troisième hypothèse, les valeurs des associations diffèrent en fonction des acteurs chargés la fonction RH et des pratiques qu'ils mettent en place. Comme l'indique la figure 3 (en page suivante), le sens de la causalité demande à être discuté. Les pratiques plus formelles et plus hiérarchiques introduites par le directeur, puis par le DRH peuvent être analysées comme une réponse à l'essoufflement du modèle communautaire 


\section{Figure 3}

Emergence de la fonction $\mathrm{RH}$ : effectifs, acteurs et valeurs

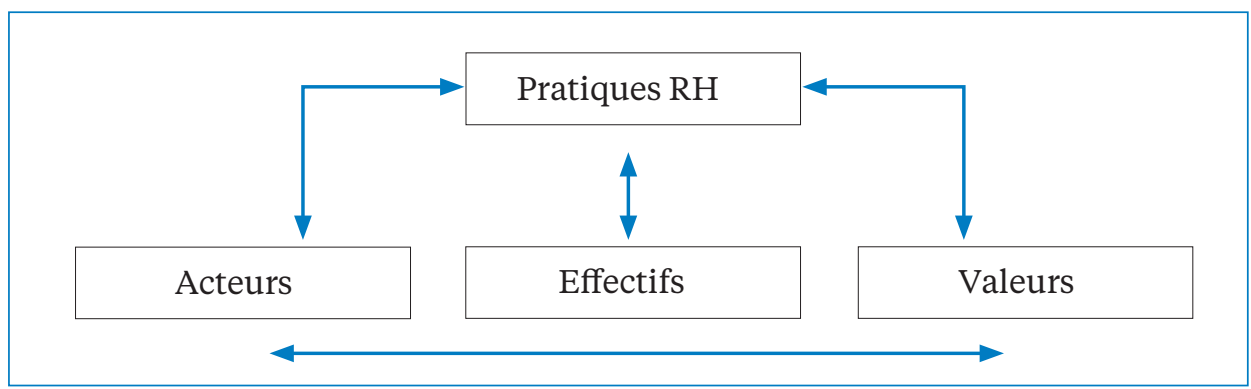

initié par le président (Greiner, 1972). Ce peut être aussi un "effet politique» des outils (Chemin, Gilbert, 2010). Leur utilisation par les acteurs constituerait un comportement les "engageant " vers de nouveaux systèmes de valeurs (Festinger, 1957).

Mais le sens de la causalité peut être inverse: les principes de liberté et d'égalité auxquels les associations sont traditionnellement attachées ne sont pas directement compatibles avec la hiérarchie sur laquelle se fondent la plupart des pratiques RH (Boncler, Valéau, 2010). Plus ses valeurs militantes seront vives, plus l'association aura tendance à résister au changement gestionnaire. D’une manière générale, l'introduction de pratiques RH plus formelles tendra à générer un clivage entre une coalition militante et une coalition gestionnaire (Védélago et al., 2004). La crise organisationnelle décrite par Greiner (1972) se double, dans le contexte associatif, d'une crise identitaire encore plus cruciale (figure 4).

\section{Figure 4}

\section{Emergence de la fonction $\mathrm{RH}$ : développement et croissance}

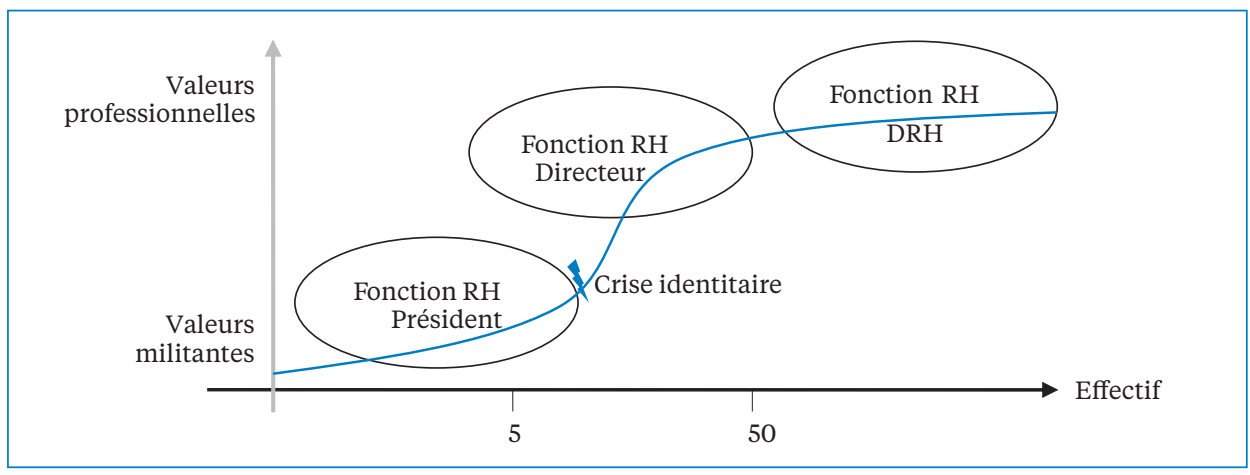

L'émergence de la fonction RH semble suivre une courbe exponentielle, de fortes tensions ralentissent son démarrage, puis elle s'accélère (figure 4). Ce phénomène s'apparente au modèle du changement social présenté par 
Mendras et Forsé (1983): une innovation au départ technique fait irréversiblement basculer la communauté dans la modernité. De façon similaire, l'introduction de nouvelles pratiques RH emporte l'organisation associative dans le monde de la rationalité gestionnaire.

\section{Conclusion}

Cet article a montré que l'émergence de la fonction RH dans les associations passe, bien avant l'arrivée d'un DRH, par le recrutement d'un directeur porteur de nouvelles motivations et de nouvelles compétences en matière d'organisation et de management. Nos données indiquaient ensuite que cette étape ne pouvait être appréhendée comme une simple rationalisation et formalisation du fonctionnement technico-économique de l'organisation à des fins d'efficacité. Elle s'accompagne, dans la plupart des cas, d'une métamorphose socioculturelle.

Les chiffres ne doivent pas faire illusion: la présente recherche reste exploratoire. Qui plus est, le processus de professionnalisation évoqué ne résulte pas d'observations longitudinales, mais de données issues de différentes associations. Nos résultats convergent cependant largement avec les constats qualitatifs développés au cours de ces dernières années. Le contexte associatif est le lieu d'une GRH traversée par des contradictions encore plus fortes que d'ordinaire. Ce dilemme entre professionnalisation de la GRH et valeurs communautaires constitue une caractéristique fondamentale de la gestion des associations. Cela dit, cette problématique se pose sans doute différemment, compte tenu des contraintes de l'activité, mais aussi de la culture, d'un secteur à l'autre et d'une organisation à l'autre.

Partant de là, nous retrouvons "l'éternelle question de la taille» (Draperi, 2012): une association peut-elle croître tout en restant un tant soit peu militante? Faut-il limiter le changement gestionnaire afin de préserver ces valeurs? Les données statistiques de cette recherche indiquent un risque, mais aucunement une fatalité. D’autres recherches plus qualitatives, à l'image de celle d'Everaere (2011) ou de Valéau (2003), pourront par la suite illustrer des façons de dépasser les tensions entre GRH professionnalisée et valeurs militantes. La gouvernance participative pourrait, par exemple, être une manière d'instituer des contre-pouvoirs (Boncler, Valéau, 2010), des espaces de médiation tels que ceux évoqués par Dussuet (2010), RichezBattesti et Oswald (2010).

$\mathrm{Au}$-delà, nous pensons que rien ne pourra se faire sans des directeurs et des DRH formés aux spécificités du mouvement associatif. Des diplômes spécifiques à la gestion des associations existent: Draperi (2011) en dénombre près de cinquante, l'Observatoire national de l'ESS (2012) en comptabilise soixante-douze, soit 1500 à 2000 diplômés par an. Avec 270000 associations employeurs et un taux de rotation des directeurs de dix ans, le besoin serait au moins de 27000 diplômés par an. Une deuxième piste consisterait à intégrer, dans l'ensemble des programmes des IAE et des écoles de commerce, des modules d'initiation aux spécificités de la gestion des associations. Cependant, le développement de ces formations nécessite "des recherches enseignables» (Lemoigne, 1990). 


\section{BIBLIOGRAPHIE}

Archambault E., Prouteau L., 2009,

« Mesurer le bénévolat », Recma, n 314 .

Bailly F., Chapelle K., Prouteau L., 2012,

"La qualité de l'emploi dans l'ESS », Recma, $\mathrm{n}^{\circ} 323$.

Barraud J., Kittel F., Moule M., 2004, La fonction RH, Paris, Dunod, deuxième édition.

Bayle E., 2010, « Gouvernement, management et performances des entreprises de l'ESS », colloque IAE et Esdes, Lyon.

Bélanger L., Petit A., Bergeron J-.L., 1983, GRH, Montréal, Gaétan Morin.

Benchenam F., Galindo G., 2009, GRH, Paris, Gualino, deuxième édition.

Biondy Y., Chatelain-Ponroy P., Eynaud P., Sponem S., 2010, «Quel modèle de gouvernance pour les associations?», Politiques et management public, vol. $27, \mathrm{n}^{\circ} 1$, p. 3-10.

Couturier R., Bodin A., Gras R., 2005, Chic version 3.5, Orléans, ARDM.

Boncler J., Valéau P., 2010, Créer et manager une association, Bruxelles, De Boeck.

CPCA, 2010, « Entrepreneuriat social et économie sociale des associations:

le débat est ouvert », 11 février.

Brabet J., 1993, «La GRH en trois modèles », in Brabet J., Repenser la GRH, Paris, Economica.

Brudney J. L., 1994,

The Jossey-Bass handbook, Los Angeles, Jossey-Bass.

Chauvière M., 2007, Trop de gestion tue le social, Paris, La Découverte.
Chemin C., Gilbert P., 2010, «L'évaluation de la performance, analyseur de la gouvernance associative ", Politiques et Management public, vol. $27, \mathrm{n}^{\circ} 1$, p. 55-78.

Combes-Joret M., Lethielleux L., 2012, " Le sens du travail à la Croix-Rouge », Recma, $\mathrm{n}^{\circ} 323$.

Davister C., 2006, La GRH en économie sociale, Louvin, chaire Cera.

Dees J. J., 1998, The meaning of social entrepreneurship, Kauffman Foundation.

Defourny J., Nyssens M., 2008, «Social enterprise in Europe », Emes, 08/01.

Defourny J., Nyssens M., 2011, «Approches européennes et américaines de l'entreprise sociale: une perspective comparative », Recma, n 319.

Delaney J. T., Huselid M. A., 1996, " The impact of HRM practices", Academy of Management Journal, vol. $39, n^{\circ} 4$, p. 171-188.

Draperi J.-F., 2010, « L'entrepreneuriat social: du marché public au public marché », Recma, $\mathrm{n}^{\circ} 316$.

Draperi J.-F., 2011, L'année sociale et solidaire, Paris, Dunod.

Draperi J.-F., 2012, « L'éternelle question de la taille », Recma, n 326 .

Dussuet A., 2010, « Un modèle associatif de régulation du travail ", Politiques et Management public, vol. 27, $\mathrm{n}^{\circ}$ 1, p. 79-96.

Everaere C., 2011. «Valeurs et pratiques de GRH dans l'économie sociale ", Revue française de gestion, $\mathrm{n}^{\circ} 217, \mathrm{p} .15-33$. 
Everaere C., Valéau P., 2012, « La GRH dans l'ESS », Le management des entreprises de l'ESS, Bruxelles, De Boeck.

Festinger L., 1957, A theory of cognitive dissonance, Evanston Row Peterson.

Fombonne J., 2001, Personnel et DRH

(France, 1830-1990), Paris, Vuibert.

Greiner L. E., 1972, « Evolution and revolution as organizations grow », Harvard Business, July, p. 37-46.

Herman R. D., 1994, Handbook of nonprofit leadership and management, Los Angeles, Jossey-Bass.

Hoarau C., Laville J.-L., 2008, Lagouvernance des associations, Toulouse, Erès.

Kaminski P., 2006, Les associations en France et leur contribution au PIB, Paris, Addes.

Laville J.-L., Sainsaulieu R., 1997, Sociologie de l'association, Paris, Desclée de Brouwer.

Le Louarn J.-Y., 2008,

Les tableaux de bord RH, Paris, Liaisons.

Lemoigne J.-L., 1990, « Epistémologies constructivistes et sciences de l'organisation ", in Alain Charles Martinet (dir.), Epistémologie et sciences de gestion, Paris, Economica.

Louart P., 1990, Cahiers de recherche, Lille, IAE Lille.

Martory B., Crozet D., 1998, Gestion RH: manuel de pilotage social, Paris, Nathan.

Mayaux F., 1996, « Noyau stratégique des associations ", thèse de doctorat, université Lyon-III.

Mendras H., Forsé M., 1983, Le changement social, Paris, Armand Colin.
Mercier E., Schmidt G., 2004, GRH, Paris, Broché.

Meyer M., Ohana M., 2010, « Justice interactionnelle, cohésion de groupe et comportements citoyens dans les entreprises sociales", Revue de GRH, n 75, p. 3-15.

\section{Mignonac K., Valéau P., Vandenberghe C.,} Gatignon A.-L., 2010,

"The commitment of volunteers ", Academy of Management Conference, Montreal.

Observatoire national de l'ESS, 2012, L'ESS dans l'enseignement supérieur, www.cncres.org.

Peaucelle J.-L., 2003, « Les disciples de Henri Fayol », Entreprise et Histoire, $\mathrm{n}^{\circ} 34$, p. 108-114.

Péretti J.-M., 2009, GRH, Paris, Vuibert, seizième édition.

Pichault F., Nizet J., 2000, Les pratiques de GRH, Paris, Seuil.

Plane J.-M., 2000,

Gestion des ressources humaines, Paris, Economica.

Richez-Battesti N., Oswald P., 2010, "Configuration de gouvernance et stratégie du faire ensemble », Politiques et management public, vol. 27, $\mathrm{n}^{\circ} 1$, p. 31-54.

Robichaud Y., McGraw E., Roger A., 2005, "L'influence des objectifs des entrepreneurs sur la performance des petites entreprises ", Revue de GRH, n 55, p. 22-35.

Rousseau F., 2007, « L'organisation militante », Recma, $\mathrm{n}^{\circ} 303$.

Salamon L. M., Anheier H. K., 1997, Defining the nonprofit sector, Manchester University Press. 
Saulquin J.-Y., 2000,

"GRH et performance des services », Revue de GRH, n 36, p. 19-31.

Sybille H., 2010, « Réponse à J.-F. Draperi », Alternatives économiques, 24 février.

Tchernonog V., 2007, Les associations en France, Paris, Addes.

Thévenet M., Dejoux C., Marbot E., Normand E., 2009,

Fonction RH, Paris, Pearson.
Valéau P., 2003,

«Différentes manières

de gérer les associations ",

Revue française

de gestion, $\mathrm{n}^{\circ} 146$, p. 9-22.

Védélago F., Valéau P., Queinnec E., 2004, "Les conflits de valeur au sein des ONG et des associations de solidarité ", Les ONG et le management, Paris, Vuibert. 